\title{
Escuelas creativas, justicia social y transdisciplinariedad: revisión sistemática
}

\author{
Veronica Violant Holz ${ }^{1}$ \\ https://orcid.org/0000-0003-2464-6845 \\ Marlene Zwierewicz ${ }^{2}$ \\ https://orcid.org/0000-0002-5840-1136 \\ Marizete Bortolanza Spessatto ${ }^{3}$ \\ https://orcid.org/0000-0003-0213-833X
}

\section{Resumen}

La educación y en consecuencia el sistema educativo necesita una verdadera reforma inclusiva que incluya el ser equitativa, gratuita y de calidad que prepare hacia la excelencia como personas ciudadanas del mundo y como profesionales proactivos. Este artículo tiene un doble objetivo, por un lado, se hace un recorrido por el surgimiento de las Escuelas Creativas, elucidando su compromiso con una educación vinculada al pensamiento complejo y a la justicia social; por el otro se realiza una revisión sistemática que analiza cómo se está dando respuesta a nivel escolar respecto a la creatividad en la práctica con acento en la justicia social y la transdiplinariedad. Se llevó a cabo una revisión en la Interfaz EBSCOhost Research en las bases de datos: ERIC; APA PsycInfo; ERIC; Teacher Reference Center; PSICODOC; APA PsycArticles con los ampliadores (aplicar materias equivalentes) y la ecuación ((creativity or creativity in education or creativity schools) and (transdisciplinary) and (social)). Se realizó la búsqueda sin poner fecha inicial y hasta el 17 de agosto de 2020. De los 36 artículos que arrojó la revisión de la literatura, 6 fueron elegibles por cumplir los criterios de inclusión. El análisis posibilitó identificar el compromiso de investigadores de varias partes del mundo, con el objetivo común de buscar formas de superar la visión descontextualizada y atomizada de la enseńanza, por medio de diferentes estrategias, como el estímulo al trabajo colaborativo, la utilización de múltiples escenarios de aprendizaje e interacción entre las asignaturas y, de estas mismas asignaturas con la realidad local y global.

1 Doctora en Psicología por la Universidad Ramon Llull, España. Profesora Titular de la Facultad de Educación de la Universitat de Barcelona (UB). Vicepresidenta de la Asociación para la Creatividad (ASOCREA). Directora del Observatorio Internacional en Pedagogía Hospitalaria. E-mail: vviolant@ub.edu.

2 Doctora en Psicología por la Universidad Federal de Santa Catarina (UFSC) y Doctora en Educación por la Universidad de Jaén (UJA). Coordinadora e investigadora del Programa de Maestría de la Universidade Alto Vale do Rio do Peixe (UNIARP). Coordinadora adjunta de la Red Internacional de Escuelas Creativas (RIEC). E-mail: marlenezwie@yahoo.com.br.

3 Doctora en Educación por la Universidad Federal de Santa Catarina (UFSC). Investigadora del Programa de Maestría ProfEPT del Instituto Federal de Educaçáo, Ciência e Tecnologia de Santa Catarina (IFSC). E-mail: marizete.spessatto@ifsc.edu.br. 
Palabras-clave: Creativity schools, Transdisciplinary education, Social justice, Sistematic review.

\section{Creative schools, social justice and transdisciplinarity: systematic review}

\section{Abstract}

Education and therefore, the educational system, needs a true inclusive reform in order to become actually equitable, free and with quality and so being able to contribute to the formation of citizens of the world and proactive professionals. This paper had a double aim, on one side, to systematize the trajectory of the Creative Schools birth, its commitment with some education which is bond to complex thought and social justice and, on the other hand, to do a systematic review that analizes how the responses are being given at the school level in relation to the creativity put into practice, with emphasis on the social justice and transdisciplinarity. A review on the Interfae EBSCOhost Research was done, under the following data base: ERIC; APA PsycInfo; ERIC; Teacher Reference Center; PSICODOC; and APA PsycArticles, with the amplifiers (applying equivalent subjects) and the equation ((creativity or creativity in education or creativity schools) and (transdisciplinary) and (social). During search, a beginning date was not selected, however, as the final date August, 17th 2020 was defined. From the thirty-six articles implicated within the review of literature, six of them were selected to follow the criteria of inclusion. The analysis could identify the commitment of the researchers of several parts of the world in seeking ways to overcome the decontextualized and atomized view of teaching, through means of different strategies, as a stimulus to the colaborative work, the use of multiple scenaries of learning and the interaction among disciplines and from these with the same local and global reality.

Keywords: Creative Schools, Transdisciplinary Education, Social justice, Systematic review.

\section{Introducción}

Enfrentar las adversidades actuales conscientes de las incertidumbres en relación con el futuro de la humanidad requiere superar referenciales de educación, pautadas en la enseñanza reproductivita, en el aprendizaje centrado en un espacio cerrado, la atomización de las asignaturas y la falta de su interacción con la realidad. Ahora, más que nunca, es imprescindible reconocer la relevancia de la creatividad en las prácticas pedagógicas.

Vivimos una época impar, motivada por consecuencias de la COVID19 que afectan un largo espectro de condiciones de la vida de la población global. Los reflejos de la pandemia incluyen problemas de salud, parte derivada de la reducción de las actividades físicas de la población confinada (AMMAR et al., 2020, VICENT et al., 2020), problemas emocionales, sociales y económicos. Además de los impactos constatados, es posible que aparezcan emergencias aun no previstas, siendo que parte de todas esas consecuencias podrá acompañar la población del planeta por muchos años, suscitando el valor de la creatividad en la superación de la adversidad en 
tiempos de crisis (TORRE, 2012a). La realidad, actual y futura, necesita personas con un alto potencial creativo e instituciones comprometidas con su desarrollo (TORRE, 2012b).

Estimular y además comprender lo que va más allá de lo visible, nos permite se capaces de proyectar y desarrollar soluciones para los problemas vivenciados. Un pensamiento capaz de, por un lado, comprender, por ejemplo, la influencia del modelo económico actual en las injusticias sociales que se amplía con la crisis generada por la pandemia y, por otro, de proponer y dinamizar soluciones que colaboren para el enfrentamiento de las adversidades vivenciadas, especialmente por la población que está más afectada por la exclusión social.

Además, la defensa de una sociedad justa pide de una educación anclada en el concepto de justicia social, con el fin de transitar de una enseñanza excluyente a un tipo de perspectiva que cree y fortalezca redes de docentes e investigadores que se comprometan con prácticas pedagógicas alineados a las necesidades en términos de justicia social. Esa es una de las condiciones que favorecieren la creación de la Red Internacional de Escuelas Creativas (RIEC) en el año 2012. En la actualidad la RIEC reúne a investigadores/as y docentes de Brasil, España, Bolivia, México y Portugal con el fin de fomentar la investigación transdisciplinaria comprometida con el bienestar individual, social y medioambiental.

Una de las iniciativas de la RIEC en la provincia de Santa Catarina Brasil consiste en el desarrollo del Programa de Formación-Acción en Escuelas Creativas en varias de sus alcaldías. Programa que se acompaña de investigaciones comprometidas en la evaluación del potencial de la transformación de la práctica pedagógica. En esta iniciativa se destaca por el espíritu de trabajo colaborativo que la mueve, por la interacción de las escuelas con la comunidad, por la pertinencia de la enseñanza y por la valoración del protagonismo docente y discente en la resolución de problemas locales que impactan globalmente.

Como instituciones que transcienden, recrían, valoran y se transforman (TORRE, 2013) las Escuelas Creativas representan una iniciativa puente para la justicia social por estimular prácticas transdisciplinarias comprometidas con demandas actuales y con las incertidumbres que ruedan el futuro de la humanidad. La constitución de una red de investigadores y docentes conectados por esa perspectiva está siendo fundamental para que diferentes contextos desarrollen prácticas pedagógicas atentas a los retos locales sin subestimar las demandas globales. 
En este artículo se hace un recorrido por el surgimiento de las Escuelas Creativas, elucidando su compromiso con una educación vinculada al pensamiento complejo y a la justicia social. Además, se presentan resultados de una revisión sistemática que analizara cómo se está dando respuesta a nivel escolar respecto a la creatividad en la práctica con acento en la justicia sociedad y la transdiplinariedad. Para el acceso a un amplio repertorio de publicaciones con esa temática, fue realizada una búsqueda por medio de la Interfaz EBSCOhost Research en bases de datos que permitirán acceder a investigaciones de diferentes partes del mundo.

\section{Justicia social, pensamiento complejo y sus conexiones con las Escuelas Creativas}

En apenas dos décadas de este nuevo siglo se ha acumulado una serie de problemas que, en forma de cascara, se sobreponen año a año sin que se observe una solución contundente. Entre ellos, Morin (2015) destaca el desreglamento ecológico, la exploración sin límites de los recursos naturaleza, la búsqueda deshumana por logros, la exclusión y la ampliación de las desigualdades sociales. Y, a pesar de iniciar el presente año (2020) con nuevas esperanzas hacia posibles soluciones para el enfrentamiento de estas temáticas, estos y los meses venideros pasaran por la gran incertidumbre del futuro de la humanidad. Esa incertidumbre se agrava, con demasía, con el surgimiento de la pandemia a nivel mundial por Covid-19 y que "[...] se ha convertido en el principal titular de salud en el mundo moderno [...] (CAMACHOCARDENOSA et al., 2020, p. 1). Además de los problemas que afectan la salud, sus efectos atingen otras áreas, entre ellas la economía, haciendo con que la humanidad atraviese en los próximos años por retos que aún no pueden se dimensionadas con total seguridad. Es en medio a esta realidad que la

[...] descualificación de la vida en común y los golpes repetidos contra el censo de colectividad contribuyen para la descualificación de la política mientras camino y espacio legítimo para la búsqueda de consensos necesarios, aunque temporarios, para la vida en común (REGO; PALÁCIOS, 2016, p. 69).

Con la debilidad del censo de colectividad la “[...] preocupación de cada uno, o cualquier uno, con las consecuencias de las acciones de cada uno, o de cualquier uno, para cualquier uno, es cada vez más un hecho menos común" (REGO; PALÁCIOS, 2019, p. 144). Debido a ese cuadro, los autores defienden la necesidad de "[...] considerar la justicia social como un 
imperativo ético laico [...] y un [...] eje en torno de lo cual se puede articular o tejido social" (REGO; PALÁCIOS, 2019, p. 141). Y es en la articulación del tejido social que la escuela ejerce una función determinante.

Se presupone que no existe justicia social si no existe justicia escolar. $\mathrm{O}$ sea, una justicia comprometida con los menos favorecidos socialmente, construyendo con ellos un proceso de escolarización que favorezca su participación, definiciones económicas y políticas (REZENDE, 2019). En este sentido, se defiende que "[..] una escuela justa y de calidad debe movilizar la justicia escolar como igualdad de tratamiento, sea en el acceso, sea en el éxito, sea en la posibilidad de permanecer en el sistema [...]", de la misma forma que favorezca una "[...] justicia diferencialista, dando más a los que más necesitan (ESTEVÃO, 2016, p. 54).

De la misma manera, la escuela justa no se desarrolla cuando se apoya en lo que Morin (1998) define como conocimiento mutilado. Un conocimiento que no posibilita la comprensión de la propia realidad de los estudiantes y, con ella, la compresión del entorno local y global. Para este autor "[...] la rarefacción del reconocimiento de los problemas complejos, la superabundancia de los saberes aislados y dispersos, parciales y fragmentarios, cuja dispersión son en sí mismos fuentes de error [...]”, configuran la existencia de un "[...] problema clave en nuestra vida de individuo, de ciudadano, de ser humano en la era planetaria, que es el problema del conocimiento [...]" (MORIN, 2015, p. 17).

En contrapartida, la realidad es compleja por ser permeada por una trama de acontecimientos, acciones, interacciones, retroacciones y determinaciones de acasos que constituyen el mundo fenoménico (MORIN, 2005). Por esto, la defensa por un pensamiento complejo y que "[...] procura demonstrar la necesidad de (re)construir un conocimiento que abarque la multidimensionalidad del hombre, de la especie y de la sociedad [...]" (SÁ, 2019, p. 22).

Para este autor, una de las tesis centrales del pensamiento complejo es la religación de los saberes y "[...] por lo tanto, la superación de la fragmentación de los conocimientos sobre el humano, la naturaleza y la sociedad". El conocimiento pertinente es justamente el conocimiento complejo, pues "[...] abarca la vida, abraza el objeto de estudio, el facto y el fenómeno [...]", ya que "[...] no hay nada o cosa o facto que pueda ser comprehendido a la luz del Pensamiento Complejo de forma aislada. Esto porque no hay nada aislado en el universo" (SÁ, 2019, p. 21).

Las Escuelas Creativas son instituciones que priman por la justicia social $y$, por esto, buscan la justicia escolar por medio de prácticas 
comprometidas con el conocimiento pertinente/complejo y con la promoción de bienestar individual, social y medioambiental. Es con esos énfasis que se comprometen con el pensar complejo.

Conceptualmente, las Escuelas Creativas son instituciones que transcienden su condición de origen, valoran los involucrados en los procesos de enseñanza y aprendizaje, recrían sus prácticas pedagógicas para que en su desarrollo ayuden a transformar la realidad mientras son también transformadas (TORRE, 2009, 2013), comprometiéndose con "[...] una educación a favor de los seres humanos y a la preservación de la biodiversidad [...]" y por la cual se comprende que "[...] somos y compartimos de una ciudadanía terrestre” (PETRAGLIA, 2013, p. 30).

Esa perspectiva tiene sus raíces involucradas en iniciativas desarrolladas en el contexto español, pero que ha se dinamizado, inicialmente, en el contexto brasileño. Esa condición es elucidada por Torre (2013) al registrar que varias han sido las iniciativas de base en el contexto espańol, entre ellas: i) un primer periodo con la realización de una serie de eventos, cursos de formación, asignaturas, proyectos de investigación y publicaciones vinculadas a la creatividad; todo esto sin, aun, mencionar la idea en la actualidad de "las Escuelas Creativas"; ii) un segundo período en el que se desarrolló el grupo de Investigación y Asesoramiento Didáctico (GIAD) dando secuencia a esas actividades, mientras también se comprometía con el desarrollo del Proyecto Estrategias Didácticas Innovadoras para la Formación Inicial de Docentes EDIFID, especialmente entre los años de 1998 e 2005; iii) un nuevo período que se constituyó a partir de 2008, cuando el Parlamento Europeo declaró 2009 como el año Europeo de la "Creatividad e Innovación”, y el Grupo GIAD elaboró el Proyecto de "Red de Escuelas Creativas" para presentar en la convocatoria da la Generalitat de Catalunya y en la convocatoria ARIE. Con el subtítulo 'hacia una escuela del siglo XXI', el documento fue elaborado con base en los principios de la sustentabilidad, ecología de los saberes e integración institucional, comprometiéndose con la superación de la fragmentación académica en forma de asignaturas. A pesar de una urgente y necesaria para la educación transformadora y de calidad, Torre (2013) afirma que la propuesta no fue aceptada por la administración, pero posibilitó llevar a cabo reflexiones sobre organizaciones educativas abiertas al cambio, que aprenden y se transforman mientras transforman su entorno; iv) una nueva etapa marcada por la organización de reuniones con el Consorcio de Educación de Catalunya (Alcaldía, Inspectoría y Departamento de Educación) para realizar una jornada o un fórum sobre Escuelas Creativas con la participación conjunta de la administración pública, la universidad y las 
escuelas, con el propósito de estimular la aproximación de saberes tradicionalmente distantes. Iniciativa que no se llevó a cabo.

Antes esas condiciones, Torre (2013, p. 147) evaluó que era necesario “[...] esperar y volver a mirar sobre otros lugares, otras tierras, otras instituciones [...] dispuestas a comprometerse con la educación transformadora”. Fue desde la interacción con profesionales del contexto brasileño que surgieron posibilidades para el desarrollo de las primeras iniciativas formativas en el sur de la provincia de Santa Catarina (Brasil) (ZWIEREWICZ, 2013).

Con ello se llevó a cabo una propuesta de formación docente y publicación, en 2009 del primero libro Escuelas Creativas, 'Uma escola para o século XXI: Escolas Criativas e resiliência na educação'. Libro que abordó conceptos que permeaban la idea de las Escuelas Creativas, donde se presentaron experiencias pedagógicas consideradas innovadoras, donde se propuso una metodológica para atender a los principios de las Escuelas Creativas y donde se concluía con reflexiones sobre la escuela pretendida (ZWIEREWICZ, 2017; ALMEIDA, 2018).

A partir de entonces surgieron posibilidades impulsadas por investigadores de diferentes Instituciones de Enseñanza Superior brasileñas en interacción con investigadores de diferentes países de Latinoamérica y de Europa, entre ellos, investigadores vinculados al Grupo de Innovación y Asesoramiento Didáctico - GIAD. Con esa interacción fue posible crear la Red Internacional de Escuelas Creativas - RIEC, durante o 'IV Fórum Internacional sobre Innovación y Creatividad: adversidad y Escuelas Creativas', realizado y la Universidad de Barcelona - UB, en junio de 2012. A cabo de 5 años, en 2017, se creó la Asociación de Escuelas Creativas - ADEC con sede en Barcelona. Asociación que tiene la posibilidad de expandirse de la mano de la RIEC, fortaleciendo así, la posibilidad de nuevas iniciativas de formación e investigación en los diferentes Núcleos RIEC.

Actualmente, la RIEC está constituida por diecinueve núcleos: RIEC UB y RIEC UAM (España), RIEC UMSA (Bolivia), RIEC UNIARP, RIEC UNIBAVE, RIEC UFG, RIEC UEG, RIEC UFT, RIEC FURB, RIEC UFAL, RIEC IFSC-SJ, RIEC MAGSUL, RIEC UNICENTRO, RIEC UNOCHAPECÓ, RIEC PUCPR, RIEC Contestado y RIEC ECOFOR (Brasil), RIEC IESF - AMARANTE (Portugal), RIEC UAP Tejupilco (México).

Por medio de los Núcleos RIEC el concepto de Escuelas Creativas se dinamiza en iniciativas que convergen con las posibilidades de cada contexto, pero alineadas a conceptos como pensamiento complexo, 
transdisciplinariedad, ecoformación, pertinencia y resiliencia. En ese proceso se estimulan, entre otras condiciones el trabajo colaborativo, estrategias didácticas innovadoras, así como, la creación y utilización de diferentes escenarios de aprendizaje en la formación docente.

Uno de los ejemplos es el Programa de Formación-Acción de Escuelas Creativas, desarrollado actualmente por el Núcleo RIEC UNIARP (Caçador - Santa Catarina). Esa propuesta se constituyó oficialmente en 2009, aunque nació de una experiencia piloto en 2008. Desde entonces, se ha desarrollado en diferentes redes municipales de enseñanza de Santa Catarina, entre ellas, Balneário Rincão, Grão Pará, Santa Rosa de Lima, São Ludgero y Paulo Lopes y también, específicamente, en algunas escuelas de la ciudad de Braço do Norte y Urussanga (ALMEIDA, 2018). Parte de las iniciativas han sido realizadas con apoyo de Núcleo RIEC UNIBAVE.

En 2018, empezaran a ser desarrolladas experiencias por el Núcleo RIEC UNIARP, implicando, especialmente docentes de las redes de los ayuntamientos de Caçador, Timbó Grande (Santa Catarina) y União da Vitória (Paraná). En todas las experiencias se utiliza como metodología de apoyo los Proyectos Creativos Ecoformadores (PCE), creados por Torre y Zwierewicz (2009) y presentados en el anterior libro enunciado ('Uma escola para o século XXI: Escolas Criativas e resiliência na educação').

Como forma de evaluar los reflejos del Programa de Formación-Acción de Escuelas Creativas son desarrolladas investigaciones vinculadas al Programa de Maestría Profesional en Educación Básica (PPGEB) de la Universidade Alto Vale do Rio do Peixe (UNIARP). Entre los resultados: i) Almeida (2018) destaca su potencial para atender necesidades formativas de los docentes porque al tener como suporte conceptos como complejidad y utilizar una metodología transdisciplinaria y ecoformadora, se conecta a realidad local sin desconsiderar las emergencias globales, utilizando en ese proceso estrategias didácticas que articulan teoría y práctica y estimulan el trabajo colaborativo; ii) Zielinski (2019), constatando que el Programa de Formación-Acción de Escuelas Creativas ha colaborado para superar la enseńanza fragmentada, por medio de actividades transdisciplinarias y ecoformadoras que estimularan a los docentes, involucrar en las clases de Educación Básica, la realidad de propia ciudad, su cultura, su producción agrícola y otras especificidades locales, mientras los alumnos estudian contenidos previstos en las diferentes asignaturas; iii) Almeida, Zwierewicz y Careño (2019, p. 104) destacan que "Trasladar las prácticas clásicas hacia prácticas transdisciplinarias y ecoformadoras puede ser tanto un reto como una necesidad". Un reto porque implica superar prácticas históricamente consolidadas y aún muy presentes en 
el contexto educacional y una posibilidad, porque existen condiciones locales y globales que piden prácticas transdisciplinarias y comprometidas con la justicia social.

Teniendo esta realidad como situación movilizadora, se visualizó la necesidad de realizar una revisión de la literatura que pudiera contestar a la siguiente pregunta: ¿Cómo lograr desarrollar a través del pensamiento creativo escuelas con una cultura más justa y transdiciplinar?

\section{Metodología}

Se ha llevado a cabo una revisión sistemática de la literatura que tuvo por objetivo analizar cómo se está dando respuesta a nivel escolar respecto a la creatividad en la práctica con acento en la justicia social y la transdiplinariedad. Este es un tipo de producción "[...] que sin ser original recopila la información más relevante sobre un tema específico” (GUIRAOGORIS; OLMEDO SALAS; FERRER FERRA, 2008, p. 1), con la finalidad de realizar el propio proceso de investigación, pero también para la actualización académica y para la aplicación de la evidencia científica y, por esto, la revisión es considerada "[...] una herramienta básica para avanzar en la práctica” (GUIRAO-GORIS, 2015, p. 4).

\section{Estrategia de búsqueda}

Para la revisión se utilizó la Interfaz EBSCOhost Research en las bases de datos: ERIC, APA PsycInfo; ERIC; Teacher Reference Center; PSICODOC; APA PsycArticles con los ampliadores (aplicar materias equivalentes) con la ecuación ((creativity or creativity in education or creativity schools) AND (transdisciplinary) AND (social)).

\section{Criterios de inclusión y exclusión y procedimiento de selección}

Con el fin de seleccionar los artículos y llegar a su lectura del texto completo, se aplicaron criterios de inclusión y exclusión, conforme descrito en el Cuadro 1. Las tres investigadoras llevaron a cabo una reunión de consenso con el fin de garantizar los criterios de selección propuestos (ANGUERA, 1990). 
Cuadro 1 - Criterios de inclusión y exclusión

\begin{tabular}{|c|c|}
\hline Criterios de inclusión & Criterios de exclusión \\
\hline $\begin{array}{l}\text { 1. Tipo de estudios: } \\
\text { 1.1. De Investigación. } \\
\text { 1.2. De intervención. } \\
\text { 1.3. Teórico. }\end{array}$ & $\begin{array}{l}\text { 1. Tipo de estudios: } \\
\text { 1.1. Ser un libro, Capítulo de } \\
\text { libro, comentario, } \\
\text { resúmenes generales de } \\
\text { congresos, tesinas, tesis } \\
\text { doctorales. }\end{array}$ \\
\hline
\end{tabular}

2. Población del estudio:

2.1. Participantes de cualquier edad.

3. Periodo y criterios de los estudios:

3.1. Publicados desde cualquier fecha hasta el 17 de agosto de 2020.

2. Estudios que no pudieran recuperarse.]

3.2. Escritos en ingles, portugués o castellano.

3.3. Cualquier país.

4. Área de interés

4.1. Educación y transdisciplinar $\mathrm{O}$

3. No estar desarrollado en el área educativa.

Educación y justicia Social.

4.2. Educación Y transdisciplinar $Y$ justicia Social.

La selección de los documentos para su lectura completa se llevó a cabo en dos fases:

Fase 1 - Todos los resúmenes de los documentos quedaron exportados. Una vez eliminados los documentos duplicados, fueron analizados aplicando los criterios presentados en la tabla 1 además de confeccionarse una tabla de análisis con los elementos relativos a los criterios de inclusión y exclusión.

Fase 2 - Las tres investigadoras, elaboraron el cuadro de análisis de la metodología, resultados y conclusiones y leyeron la totalidad de los artículos seleccionados.

Cabe comentar que inicialmente se llevaron a cabo otras búsquedas que acogieran conceptos más específicos: creatividad y educación, didáctica, ecoformación, educación transdisciplinar, justicia social, justicia social en educación, sin obtener resultados o resultados muy pobres. 


\section{Resultados}

De los 36 artículos que arrojó la revisión, estos fueron analizados y aplicados los criterios de inclusión y exclusión, dando como resultado el análisis del texto de completo de 13 artículos (en la tabla 2, se muestra el análisis llevado a cabo en esta primera fase de selección. Finalmente, por diferentes motivos 7 artículos fueron eliminados: 1) ser una tesis doctoral (3 artículos); 2) no tener posibilidad de leer el texto completo (3 artículos) y; 3) tratarse de un libro (1 artículo). Fueron elegibles para su análisis cualitativo, un total de 6 artículos, tal y como muestra el diagrama de flujo (Figura 1) de PRISMA (MOHER et al, 2009).

Figura 1 - Diagrama de flujo

PRISMA 2009 Flow Diagram
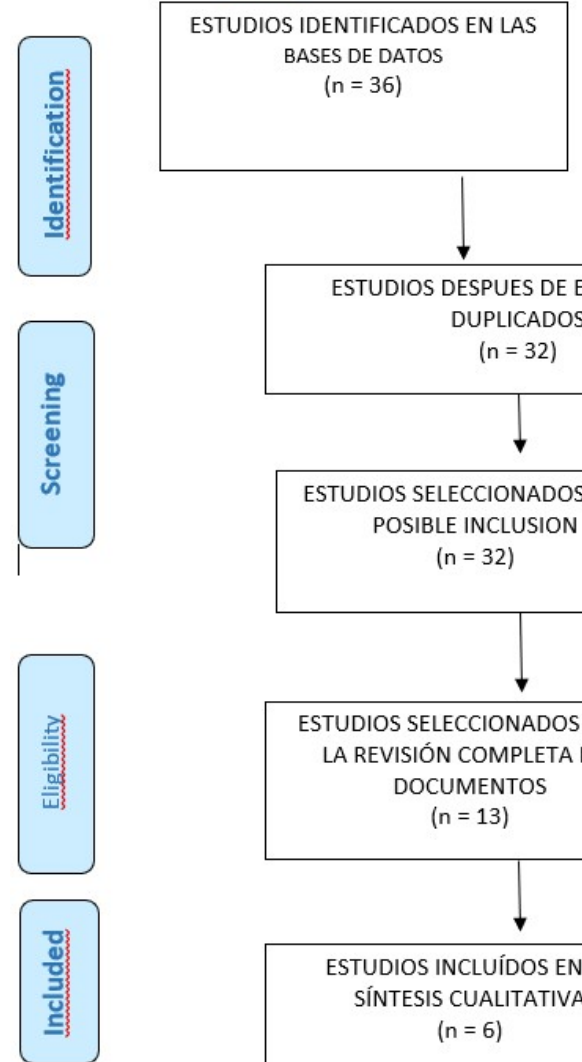

ESTUDIOS DESPUES DE EXCLUIR LOS DUPLICADOS $(\mathrm{n}=32)$
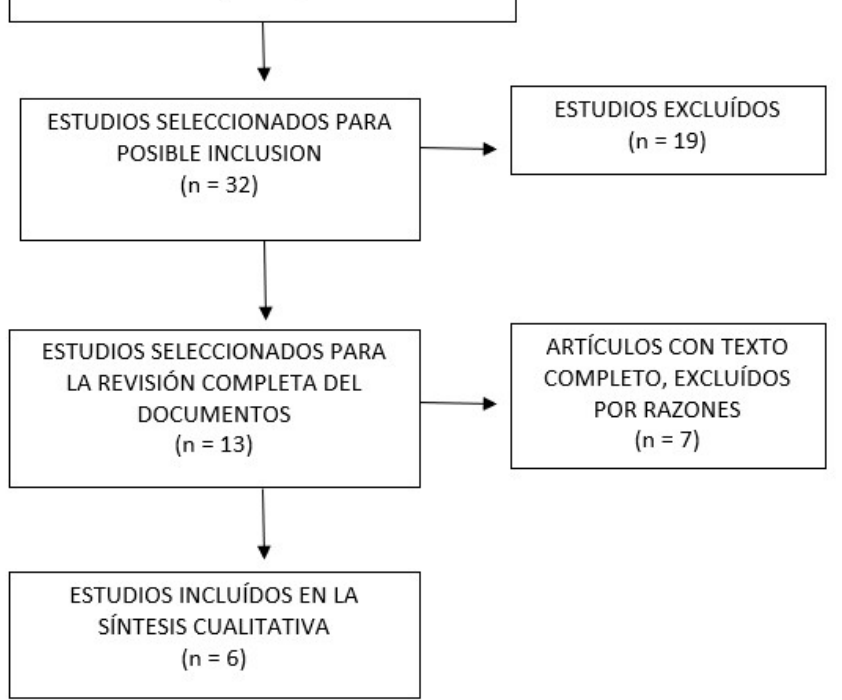

ARTÍCULOS CON TEXTO COMPLETO, EXCLUÍDOS POR RAZONES $(n=7)$ 
El Cuadro 2, a seguir, presenta la descripción hecha con base en la lectura de los resúmenes de los 32 artículos seleccionados en la primera etapa de la investigación.

Cuadro 2 - Conceptos clave de las publicaciones seleccionados para su análisis como elegibles (32 artículos)

\begin{tabular}{|c|c|c|}
\hline Autor/Ańo & Título & Conceptos clave \\
\hline Adra (2017) & $\begin{array}{l}\text { The states of presence and insight } \\
\text { in the painting process }\end{array}$ & Creatividad; Neurociencias; Arte \\
\hline Ayvaz (2018) & $\begin{array}{l}\text { Generating creativity in teams: An } \\
\text { exploratory case study. }\end{array}$ & $\begin{array}{l}\text { Innovación; } \\
\text { Administración }\end{array}$ \\
\hline $\begin{array}{l}\text { Association } \\
\text { for the } \\
\text { Advancement } \\
\text { of } \\
\text { Computing } \\
\text { in Education } \\
(2012)\end{array}$ & E-Learn 2012 & $\begin{array}{l}\text { Enfoque interdisciplinario; } \\
\text { Educación multicultural; Educación } \\
\text { corporativa. }\end{array}$ \\
\hline $\begin{array}{l}\text { Charyton } \\
(2015)\end{array}$ & $\begin{array}{l}\text { Creativity and innovation among } \\
\text { science and art: A discussion of } \\
\text { the two cultures. }\end{array}$ & Creatividad; Ciencia; Arte \\
\hline $\begin{array}{l}\text { Dehnad } \\
(2019)\end{array}$ & $\begin{array}{l}\text { Sustainable Transdisciplinary } \\
\text { Future for English Majors in Iran } \\
\text { by Implementing a New } \\
\text { Paradigm }\end{array}$ & $\begin{array}{lr}\text { Creatividad; } & \text { Abordaje } \\
\text { Interdisciplinario; } & \text { Conciencia } \\
\text { Cultural. } & \end{array}$ \\
\hline $\begin{array}{l}\text { DePalo e } \\
\text { Brennan, } \\
(2004)\end{array}$ & $\begin{array}{l}\text { Spiritual Caregiving for Older } \\
\text { Adults: A Perspective from } \\
\text { Clinical Practice. }\end{array}$ & $\begin{array}{l}\text { Cuidado espiritual; Holistico; } \\
\text { Intervencion. }\end{array}$ \\
\hline $\begin{array}{l}\text { Fonseca } \\
(2010)\end{array}$ & $\begin{array}{l}\text { Metodologías participativas, } \\
\text { subjetividades expandidas y } \\
\text { transdisciplinariedad. }\end{array}$ & $\begin{array}{l}\text { Programas de intervención; } \\
\text { Creatividad; } \\
\text { Cooperación. }\end{array}$ \\
\hline $\begin{array}{l}\text { Guyotte et al. } \\
(2015)\end{array}$ & $\begin{array}{l}\text { Steam as Social Practice: } \\
\text { Cultivating Creativity in } \\
\text { Transdisciplinary Spaces. }\end{array}$ & $\begin{array}{l}\text { Creatividad; Enfoque } \\
\text { interdisciplinario; Justicia social. }\end{array}$ \\
\hline $\begin{array}{l}\text { Gülerce } \\
(2017)\end{array}$ & $\begin{array}{l}\text { Conceptual and conditional } \\
\text { (im)possibilities of creative } \\
\text { theorizing of creativity and } \\
\text { culture: Critical reflections from } \\
\text { Turkey toward globally } \\
\text { transformative praxis. }\end{array}$ & $\begin{array}{lr}\text { Creatividad; } & \text { Cultura } \\
\text { (Antropológica) } & \text { Globalización; } \\
\text { Psicología. } & \end{array}$ \\
\hline $\begin{array}{l}\text { Hall et al. } \\
(2008)\end{array}$ & $\begin{array}{l}\text { Moving the science of team } \\
\text { science forward: Collaboration } \\
\text { and creativity }\end{array}$ & $\begin{array}{l}\text { Colaboración; creatividad; salud } \\
\text { pública. }\end{array}$ \\
\hline Hauk (2015) & $\begin{array}{l}\text { Gaia e/mergent: } \quad \text { Earth } \\
\text { regenerative education catalyzing }\end{array}$ & Empatía; Innovación; Creatividad. \\
\hline
\end{tabular}




\begin{tabular}{|c|c|c|}
\hline & empathy, creativity, and wisdom. & \\
\hline $\begin{array}{l}\text { Helmick } \\
(2019)\end{array}$ & $\begin{array}{l}\text { Extraordinary expressions of trust } \\
\text { and vulnerability: An arts- } \\
\text { informed transdisciplinary } \\
\text { journey through blended } \\
\text { landscapes of art education and } \\
\text { art therapy. }\end{array}$ & $\begin{array}{l}\text { Educación artística; Terapia artística; } \\
\text { Creatividad. }\end{array}$ \\
\hline $\begin{array}{l}\text { Henriksen } \\
(2012)\end{array}$ & $\begin{array}{l}\text { We teach who we are: Creativity } \\
\text { and trans-disciplinary thinking in } \\
\text { the practices of accomplished } \\
\text { teachers. }\end{array}$ & $\begin{array}{l}\text { Creatividad; } \quad \text { Pensamiento } \\
\text { transdisciplinar; } \\
\text { experiencia. }\end{array}$ \\
\hline $\begin{array}{l}\text { Henrickson } \\
(2016)\end{array}$ & $\begin{array}{l}\text { Adventure, inquiry, and } \\
\text { technology as driving forces in } \\
\text { sustainability education. }\end{array}$ & $\begin{array}{l}\text { Educación; Tecnología; Desarrollo } \\
\text { sostenible. }\end{array}$ \\
\hline $\begin{array}{l}\text { Jackson } \\
(2018)\end{array}$ & $\begin{array}{l}\text { The implications of self-creation } \\
\text { and self-care in higher education: } \\
\text { A transdisciplinary inquiry. }\end{array}$ & $\begin{array}{l}\text { Auto-creación; Educación Superior; } \\
\text { Desarrollo curricular. }\end{array}$ \\
\hline $\begin{array}{l}\text { Kahhale, e } \\
\text { Esper (2014) }\end{array}$ & \begin{tabular}{llr} 
Novas & \multicolumn{2}{r}{ possibilidades } \\
metodológica: a quebra & dos \\
paradigmas & qualitativo & e \\
quantitativo em & psicologia. &
\end{tabular} & $\begin{array}{l}\text { Investigación } \\
\text { Metodología; } \\
\text { psicológica. }\end{array}$ \\
\hline $\begin{array}{l}\text { Keane e } \\
\text { Keane (2016) }\end{array}$ & STEAM by Design. & $\begin{array}{l}\text { Educación STEM; } \\
\text { artística; Creatividad. }\end{array}$ \\
\hline $\begin{array}{l}\text { LaDuca et al. } \\
(2019)\end{array}$ & $\begin{array}{l}\text { A } \\
\text { Collaboration and Innovation } \\
\text { Education model and Experience. }\end{array}$ & $\begin{array}{l}\text { Enfoque interdisciplinario; } \\
\text { Innovación Educativa; Creatividad. }\end{array}$ \\
\hline $\begin{array}{l}\text { Marchiori e } \\
\text { Oliveira } \\
(2009)\end{array}$ & $\begin{array}{l}\text { Perspectives, challenges, and } \\
\text { future directions for } \\
\text { organizational communication } \\
\text { research in Brazil. }\end{array}$ & $\begin{array}{lr}\text { Organizaciones de } & \begin{array}{r}\text { negocios; } \\
\text { Comunicación; }\end{array} \\
\text { multidisciplinarias } & y \\
\text { transdisciplinarias con áreas como la } \\
\text { administración. }\end{array}$ \\
\hline $\begin{array}{l}\text { Mercieca } \\
(2012)\end{array}$ & $\begin{array}{l}\text { I'm not so sure, Miss'. The } \\
\text { concept of uncertainty and } \\
\text { dramatherapy practice within the } \\
\text { context of transdisciplinary work } \\
\text { in an educational setting. }\end{array}$ & $\begin{array}{l}\text { Dramaterapia; } \\
\text { transdisciplinario; } \\
\text { educativos. }\end{array}$ \\
\hline $\begin{array}{l}\text { Montuori e } \\
\text { Donnelly } \\
(2016)\end{array}$ & $\begin{array}{l}\text { The creativity of culture and the } \\
\text { culture of creativity research: The } \\
\text { promise of integrative } \\
\text { transdisciplinarity. }\end{array}$ & $\begin{array}{l}\text { Creatividad; Cultura; Investigación } \\
\text { transdisciplinar. }\end{array}$ \\
\hline $\begin{array}{l}\text { Penaluna, } \\
\text { Coates e } \\
\text { Penaluna } \\
(2010)\end{array}$ & $\begin{array}{l}\text { Creativity-Based Assessment and } \\
\text { Neural Understandings: A } \\
\text { Discussion and Case Study } \\
\text { Analysis. }\end{array}$ & $\begin{array}{l}\text { Creatividad; Emprendimiento; } \\
\text { Procesos cognitivos. }\end{array}$ \\
\hline Picucci & Ritual as resource: Health and & Cambio de cultura; Comunidades; \\
\hline
\end{tabular}




\begin{tabular}{|c|c|c|}
\hline$(2017)$ & $\begin{array}{l}\text { transformation in the twenty-first } \\
\text { century. }\end{array}$ & Interacción de tecnología humana. \\
\hline Prater (1997) & $\begin{array}{l}\text { New Hopes, New Horizons: The } \\
\text { Challenges of Diversity in } \\
\text { Education. Proceedings of the } \\
\text { Biennial International }\end{array}$ & $\begin{array}{l}\text { Estrategias de evaluación para } \\
\text { personas con autismo; Desarrollar } \\
\text { estándares para profesionales que } \\
\text { trabajan con estudiantes con } \\
\text { excepcionalidades; Enseñanza } \\
\text { cooperativa y creación de equipos } \\
\text { eficaces. }\end{array}$ \\
\hline $\begin{array}{l}\text { Reason et al. } \\
(2016)\end{array}$ & $\begin{array}{l}\text { Spectators' aesthetic experience of } \\
\text { sound and movement in dance } \\
\text { performance: A transdisciplinary } \\
\text { investigation. }\end{array}$ & $\begin{array}{l}\text { Estética; } \\
\text { Transdisciplinariedad. }\end{array}$ \\
\hline $\begin{array}{l}\text { Roels e Van } \\
\text { Petegem } \\
(2016)\end{array}$ & $\begin{array}{l}\text { Transdisciplinary dimensions in } \\
\text { the composing activities of } \\
\text { children: Transfer of strategies } \\
\text { and transformation of knowledge. }\end{array}$ & $\begin{array}{l}\text { Expresión Visual; Enseñanza niños; } \\
\text { Música y dibujos. }\end{array}$ \\
\hline $\begin{array}{l}\text { Rosencrans } \\
(2020)\end{array}$ & $\begin{array}{l}\text { Narratives of ingenuity: Using } \\
\text { coworking space stories to see } \\
\text { systems change. }\end{array}$ & $\begin{array}{l}\text { Creatividad; Imaginación; } \\
\text { Organizaciones de co-working. }\end{array}$ \\
\hline $\begin{array}{l}\text { Stroebe } \\
(2006)\end{array}$ & $\begin{array}{l}\text { Interdisciplinary Research and } \\
\text { Creativity. }\end{array}$ & $\begin{array}{l}\text { Creatividad; Investigación } \\
\text { interdisciplinaria; Psicología Social. }\end{array}$ \\
\hline $\begin{array}{l}\text { Thompson e } \\
\text { Kreuter } \\
(2014)\end{array}$ & $\begin{array}{l}\text { Using written narratives in public } \\
\text { health practice: A creative writing } \\
\text { perspective. }\end{array}$ & $\begin{array}{l}\text { Enfermedad crónica; Escritura } \\
\text { creativa; Servicios de salud pública. }\end{array}$ \\
\hline $\begin{array}{l}\text { Vaughan et } \\
\text { al. (2019) }\end{array}$ & $\begin{array}{l}\text { Developing creativity to enhance } \\
\text { human potential in sport: A } \\
\text { wicked } \\
\text { challenge. }\end{array}$ & $\begin{array}{l}\text { Investigación transdisciplinar; } \\
\text { Limitaciones socioculturales; Formas } \\
\text { de vida; Fútbol. }\end{array}$ \\
\hline $\begin{array}{l}\text { Whitbread } \\
(2018)\end{array}$ & $\begin{array}{l}\text { Cultural and arts education } \\
\text { policies in Hong Kong: Two } \\
\text { wings of the same bird? }\end{array}$ & $\begin{array}{l}\text { Artes; Cultura de cambio; Política } \\
\text { Educativa. }\end{array}$ \\
\hline West (2006) & $\begin{array}{l}\text { Dissent in Teams and } \\
\text { Organizations: Lessons for Team } \\
\text { Innovation and Empowerment. }\end{array}$ & $\begin{array}{lr}\text { Psicología Social; } & \text { Cambio } \\
\text { Organizacional; } & \text { Psicología } \\
\text { Organizacional. } & \end{array}$ \\
\hline
\end{tabular}

Fonte: Adra (2017), Ayvaz (2018), Association for the Advancement of Computing in Education (2012), Charyton (2015), Dehnad (2019), DePalo e Brennan, (2004), Fonseca (2010), Guyotte et al. (2015), Gülerce (2016), Hall et al. (2008), Hauk (2015), Helmick (2019), Henriksen (2012), Henrickson (2016), Jackson (2018), Kahhale; Esper (2014), Keane e Keane (2016), LaDuca et al. (2019), Marchiori; Oliveira (2009), Mercieca (2012), Montuori; Donnelly (2016), Penaluna, Coates e Penaluna (2010), Picucci (2017), Prater (1997), Reason et al. (2016), Roels e Van Petegem (2016), Rosencrans (2020), Stroebe (2006), 
Thompson; Kreuter (2014), Vaughan et al. (2019), Whitbread (2018) e West (2006). Nota. El título se ha dejado en el idioma que aparece.

A pesar de no acotar los años en la ecuación de búsqueda, los 32 estudios que arrojó la ecuación fueron publicados entre los años de 2010 y 2020, evidenciando que las investigaciones sobre el tema todavía son recientes tanto en términos de desarrollo de propuestas prácticas en las escuelas, cuanto en recogida de datos, sistematización y socialización de resultados. Entre los más recientes están los de: Dehnad (2020), Helmick (2019) y Rosencrans (2020). También es importante destacar que la diversidad territorial de las publicaciones, provenientes de países como Brasil, Canadá, China, Colombia, Estados Unidos, Irán e Inglaterra.

La temática de la transdisciplinariedad está en prácticamente la mitad de los títulos, articulándose con innovación (1), educación superior (32), ambiente escolar $(14,16)$, además de vincularse a estudios en diferentes lenguajes como las danzas (24/25) y la lengua inglesa (11); apareciendo también en otras publicaciones al articular los debates acerca de la integración entre ciencia y arte (4), por ejemplo, y a la práctica docente, como en el artículo 36, que trata del pensamiento transdisciplinar en práctica del profesorado.

Los estudios arrojados en esta primera etapa también ponen en evidencia la temática creatividad en investigaciones que tratan del deporte (8), de las políticas de educación cultural y artística (7), de la educación en las organizaciones (9), salud pública, (35) e espiritualidad (26/27). La creatividad articulada con la práctica transformadora (3).

De los 32 artículos seleccionados y leídos sus resúmenes, finalmente 6 fueron elegibles para su lectura y análisis del texto completo por tratarse de artículos que daban razón a la 'Educación y transdisciplinar' y 'Educación y justicia social' (Cuadro 3).

Cuadro 3 - Objetivos, metodología, resultados y conclusiones de las publicaciones seleccionadas

\begin{tabular}{|c|c|c|c|c|c|}
\hline Autor & Título & Objetivo & Metodología & Resultados & Conclusiones \\
\hline $\begin{array}{c}\text { Fonseca } \\
(2010)\end{array}$ & $\begin{array}{l}\text { Metodologías } \\
\text { participativas, } \\
\text { subjetividades } \\
\text { expandidas y } \\
\text { transdisciplinarie } \\
\text { dad }\end{array}$ & $\begin{array}{l}\text { Comprender la } \\
\text { pertinencia } \\
\text { social de la } \\
\text { construcción de } \\
\text { metodologías } \\
\text { participativas y } \\
\text { de creación de } \\
\text { acontecimientos } \\
\text { transdisciplinari } \\
\text { os para el } \\
\text { trabajo }\end{array}$ & $\begin{array}{l}\text { Revisión del } \\
\text { estado de } \\
\text { prácticas de } \\
\text { intervención } \\
\text { social } \\
\text { interdisciplina } \\
\text { ria con } \\
\text { jóvenes en } \\
\text { Iberoamérica } \\
\text { y una } \\
\text { documentació }\end{array}$ & $\begin{array}{l}\text { Los clubes de } \\
\text { arte, ciencia y } \\
\text { tecnología } \\
\text { (prototipos } \\
\text { experimentales } \\
\text { para el rediseño } \\
\text { de procesos } \\
\text { organizacionale } \\
\text { s) se } \\
\text { constituyen } \\
\text { como espacios }\end{array}$ & $\begin{array}{l}\text { La invención de } \\
\text { rutas } \\
\text { metodológicas } \\
\text { ofrece } \\
\text { posibilidades } \\
\text { para activar } \\
\text { procesos de } \\
\text { formación } \\
\text { interdisciplinari } \\
\text { os, generar } \\
\text { formas de }\end{array}$ \\
\hline
\end{tabular}




\begin{tabular}{|c|c|c|c|c|c|}
\hline & & $\begin{array}{l}\text { comunitario } \\
\text { con jóvenes en } \\
\text { el ámbito del } \\
\text { arte, la ciencia y } \\
\text { la tecnología. }\end{array}$ & $\begin{array}{l}\text { n teórica en } \\
\text { que se } \\
\text { obtuvieran } \\
\text { algunos } \\
\text { referentes que } \\
\text { orientaran el } \\
\text { trabajo. Un } \\
\text { segundo } \\
\text { momento } \\
\text { consistió en el } \\
\text { trabajo de } \\
\text { formación que } \\
\text { desarrolló el } \\
\text { equipo asesor } \\
\text { con el } \\
\text { conjunto de } \\
\text { tutores. } \\
\text { construcción } \\
\text { de una ruta } \\
\text { metodológica- } \\
\text { pedagógica } \\
\text { por localidad. }\end{array}$ & $\begin{array}{l}\text { para la } \\
\text { creatividad } \\
\text { social; son un } \\
\text { ejemplo } \\
\text { importante de } \\
\text { escenarios } \\
\text { educativos en } \\
\text { que se articulan } \\
\text { los afectos, las } \\
\text { necesidades y } \\
\text { los intereses de } \\
\text { los jóvenes a } \\
\text { partir de } \\
\text { proyectos } \\
\text { creativos. En } \\
\text { los clubes se les } \\
\text { sigue la huella } \\
\text { a los jóvenes, se } \\
\text { les abren rutas } \\
\text { de la potencia y } \\
\text { para un } \\
\text { reconocimiento } \\
\text { de su } \\
\text { singularidad y } \\
\text { su alteridad. }\end{array}$ & $\begin{array}{l}\text { pensamiento y } \\
\text { de abstracción } \\
\text { frente a lo que } \\
\text { se piensa y se } \\
\text { hace. } \\
\text { Los clubes, } \\
\text { desde la } \\
\text { perspectiva } \\
\text { metodológica y } \\
\text { pedagógica, son } \\
\text { una posibilidad } \\
\text { formativa para } \\
\text { los jóvenes, en } \\
\text { la medida que } \\
\text { se constituyen } \\
\text { como referentes } \\
\text { socializadores } \\
\text { de } \\
\text { experimentació } \\
\text { n colectiva, } \\
\text { como el } \\
\text { territorio y el } \\
\text { saber. }\end{array}$ \\
\hline $\begin{array}{l}\text { Guyott } \\
\text { e } \text { et al. } \\
(2015)\end{array}$ & $\begin{array}{c}\text { Steam as Social } \\
\text { Practice: } \\
\text { Cultivating } \\
\text { Creativity in } \\
\text { Transdisciplinary } \\
\text { Spaces }\end{array}$ & $\begin{array}{l}\text { A partir de la } \\
\text { descripción de } \\
\text { una experiencia } \\
\text { colaborativa } \\
\text { entre arte- } \\
\text { educación, } \\
\text { ingeniería y } \\
\text { arquitectura } \\
\text { paisajística, } \\
\text { conceptuar el } \\
\text { STEAM como } \\
\text { una práctica } \\
\text { social que } \\
\text { reflete las } \\
\text { preocupaciones } \\
\text { con la } \\
\text { involucración } \\
\text { de la } \\
\text { comunidad y la } \\
\text { sostenibilidad } \\
\text { ecológica, } \\
\text { transcendiendo } \\
\text { los objetivos } \\
\text { tradicionales de } \\
\text { la educación } \\
\text { atomizada en } \\
\text { asignaturas para } \\
\text { abordar } \\
\text { cuestiones de la } \\
\text { práctica social. }\end{array}$ & $\begin{array}{l}\text { Descripción } \\
\text { de experiencia } \\
\text { colaborativa } \\
\text { transdisciplina } \\
\text { r. }\end{array}$ & $\begin{array}{l}\text { El foco en } \\
\text { desafíos } \\
\text { complejos de } \\
\text { diseńo de la } \\
\text { sostenibilidad } \\
\text { local y global } \\
\text { funcionó como } \\
\text { un vehículo a } \\
\text { través del cual } \\
\text { fue posible } \\
\text { pensar por } \\
\text { medio de } \\
\text { materiales, } \\
\text { considerar el } \\
\text { público y se } \\
\text { involucrar con } \\
\text { la comunidad. }\end{array}$ & $\begin{array}{l}\text { La Educación } \\
\text { STEAM tiene } \\
\text { un fuerte } \\
\text { potencial para } \\
\text { la formación de } \\
\text { los } \\
\text { profesionales } \\
\text { comprometidos } \\
\text { con el contexto } \\
\text { social. } \\
\text { Estudiantes } \\
\text { entendieran el } \\
\text { papel de la } \\
\text { Educación } \\
\text { STEAM como } \\
\text { una experiencia } \\
\text { que permite el } \\
\text { hacer (y no } \\
\text { solamente } \\
\text { recibir } \\
\text { informaciones } \\
\text { terminadas en } \\
\text { clase). }\end{array}$ \\
\hline
\end{tabular}




\begin{tabular}{|c|c|c|c|c|c|}
\hline $\begin{array}{c}\text { Keane e } \\
\text { Keane } \\
(2016)\end{array}$ & $\begin{array}{l}\text { STEAM by } \\
\text { Design }\end{array}$ & $\begin{array}{l}\text { Presentar } \\
\text { posibilidades } \\
\text { para estimular } \\
\text { el proceso de } \\
\text { autonomía de } \\
\text { los estudiantes } \\
\text { en la búsqueda } \\
\text { de } \\
\text { conocimiento } \\
\text { escolar y del } \\
\text { mundo. }\end{array}$ & $\begin{array}{l}\text { Estudio de } \\
\text { caso } \\
\text { realizado con } \\
\text { cuatro talleres } \\
\text { y escuelas de } \\
\text { la Enseńanza } \\
\text { Fundamental } \\
\text { y Media. }\end{array}$ & $\begin{array}{l}\text { Los talleres con } \\
\text { estudiantes de } \\
\text { la Enseńanza } \\
\text { Fundamental y } \\
\text { Media } \\
\text { generaron } \\
\text { nuevas formas } \\
\text { de aprender. } \\
\text { Después del } \\
\text { workshop, los } \\
\text { alumnos } \\
\text { relataron que } \\
\text { les gustó haber } \\
\text { tenido la } \\
\text { oportunidad de } \\
\text { usar } \\
\text { herramientas } \\
\text { simultaneas } \\
\text { para } \\
\text { interacción con } \\
\text { objetos, } \\
\text { tecnologías } \\
\text { espacios y } \\
\text { ambientes. } \\
\text { Conectando } \\
\text { escalas de } \\
\text { diseńo } \\
\text { afectadas por } \\
\text { tópicos, los } \\
\text { alumnos han } \\
\text { sido } \\
\text { presentados al } \\
\text { pensamiento } \\
\text { sistémico y al } \\
\text { pensamiento } \\
\text { relacional. }\end{array}$ & $\begin{array}{l}\text { El uso de } \\
\text { recursos online } \\
\text { ayuda a } \\
\text { expandir las } \\
\text { oportunidades } \\
\text { de diseńo y } \\
\text { construir } \\
\text { informaciones, } \\
\text { tomando } \\
\text { decisiones } \\
\text { permite al } \\
\text { alumno } \\
\text { procesar nuevas } \\
\text { informaciones a } \\
\text { lo largo del } \\
\text { tiempo. } \\
\text { El “STEAM by } \\
\text { Design" crea } \\
\text { contextos para } \\
\text { atención } \\
\text { focalizada, } \\
\text { funcionando } \\
\text { como suporte } \\
\text { de habilidades y } \\
\text { experiencia y } \\
\text { permite el } \\
\text { desarrollo de la } \\
\text { imaginación en } \\
\text { las escuelas con } \\
\text { nuevas formas } \\
\text { de conocer y } \\
\text { aprender a } \\
\text { hacer. } \\
\text { a }\end{array}$ \\
\hline $\begin{array}{l}\text { LaDuca } \\
\text { et al. } \\
(2019)\end{array}$ & $\begin{array}{c}\text { A } \\
\text { Transdisciplinary } \\
\text { collaboration } \\
\text { and Innovation } \\
\text { Education } \\
\text { Model and } \\
\text { Experience }\end{array}$ & $\begin{array}{l}\text { Presentar } \\
\text { resultados de la } \\
\text { implementación } \\
\text { de un modelo } \\
\text { transdisciplinar } \\
\text { de educación, } \\
\text { vinculando } \\
\text { diferentes } \\
\text { asignaturas a } \\
\text { través de un } \\
\text { tema común y } \\
\text { dentro de un } \\
\text { espacio común. }\end{array}$ & $\begin{array}{l}\text { Fue utilizado } \\
\text { un } \\
\text { cuestionario } \\
\text { con preguntas } \\
\text { demográficas } \\
\text { sobre los } \\
\text { alumnos que } \\
\text { participaran } \\
\text { de la } \\
\text { propuesta y } \\
\text { preguntas } \\
\text { sobre la } \\
\text { experiencia, } \\
\text { además de } \\
\text { entrevista. }\end{array}$ & $\begin{array}{l}\text { El } \\
\text { reconocimiento } \\
\text { de los alumnos } \\
\text { de que la } \\
\text { experiencia ha } \\
\text { mejorado su } \\
\text { capacidad de } \\
\text { colaborar en } \\
\text { un ambiente } \\
\text { transdisciplinar } \\
\text { y esto impactó } \\
\text { significativame } \\
\text { nte en sus } \\
\text { aspiraciones de } \\
\text { carrera } \\
\text { profesional. } \\
\text { Algunos de los } \\
\text { alumnos } \\
\text { reconocieron } \\
\text { que la } \\
\text { experiencia }\end{array}$ & $\begin{array}{l}\text { La } \\
\text { involucración } \\
\text { de los } \\
\text { estudiantes de } \\
\text { diferentes } \\
\text { asignaturas } \\
\text { constituyo una } \\
\text { oportunidad de } \\
\text { compartir } \\
\text { perspectivas } \\
\text { con otras } \\
\text { especialidades. } \\
\text { La empatía } \\
\text { generada por } \\
\text { las perspectivas } \\
\text { de los otros } \\
\text { influenció } \\
\text { claramente el } \\
\text { producto de la } \\
\text { mayoría de los } \\
\text { alumnos. }\end{array}$ \\
\hline
\end{tabular}




\begin{tabular}{|c|c|c|c|c|c|}
\hline & & & & $\begin{array}{l}\text { mejoró incluso } \\
\text { su capacidad de } \\
\text { crear. }\end{array}$ & $\begin{array}{l}\text { Lo que más se } \\
\text { destaca en la } \\
\text { experiencia es la } \\
\text { disponibilidad } \\
\text { de un espacio } \\
\text { flexible para el } \\
\text { aprendizaje es } \\
\text { crucial para } \\
\text { promocionar la } \\
\text { colaboración, } \\
\text { mientras la } \\
\text { libertad de cada } \\
\text { estudiante para } \\
\text { la apropiación } \\
\text { de su } \\
\text { aprendizaje e } \\
\text { ideación } \\
\text { Entre las } \\
\text { sugerencias, se } \\
\text { indican la } \\
\text { publicación de } \\
\text { la inmersión } \\
\text { más profunda } \\
\text { de los } \\
\text { estudiantes en } \\
\text { la comunidad y } \\
\text { el cuidado con } \\
\text { la sobrecarga de } \\
\text { informaciones. }\end{array}$ \\
\hline $\begin{array}{l}\text { Penalu } \\
\text { na, } \\
\text { Coates } \\
\text { e } \\
\text { Penalu } \\
\text { na } \\
(2010)\end{array}$ & $\begin{array}{c}\text { Creativity-Based } \\
\text { Assessment and } \\
\text { Neural } \\
\text { Understandings: } \\
\text { A Discussion } \\
\text { and Case Study } \\
\text { Analysis }\end{array}$ & $\begin{array}{l}\text { la } \\
\text { Presentar } \\
\text { pedagogías } \\
\text { basadas en } \\
\text { diseńo y } \\
\text { destacar } \\
\text { abordajes } \\
\text { cognitivos que } \\
\text { desarrollan } \\
\text { mentalidades } \\
\text { innovadoras, } \\
\text { observando } \\
\text { posibilidades de } \\
\text { uso en la } \\
\text { educación para } \\
\text { el } \\
\text { emprendeduris } \\
\text { mo. }\end{array}$ & $\begin{array}{l}\text { Revisión de } \\
\text { literatura y } \\
\text { estudio de } \\
\text { caso. }\end{array}$ & $\begin{array}{l}\text { Negocios y } \\
\text { creatividad se } \\
\text { combinan, } \\
\text { proporcionand } \\
\text { o una } \\
\text { oportunidad } \\
\text { los alumnos } \\
\text { aprendieren } \\
\text { como innovar } \\
\text { y ofrecer } \\
\text { soluciones para } \\
\text { problemas } \\
\text { desafiadores, } \\
\text { relacionados a } \\
\text { los negocios. } \\
\text { Los alumnos se } \\
\text { tornan } \\
\text { intérpretes de } \\
\text { las } \\
\text { aspiraciones, } \\
\text { servicios y } \\
\text { productos de } \\
\text { sus clientes. } \\
\text { Los alumnos } \\
\text { relatan que las } \\
\text { ideas acerca de } \\
\text { las creaciones } \\
\text { llegan a la } \\
\text { mente cuando }\end{array}$ & $\begin{array}{l}\text { Existe una } \\
\text { escasez de } \\
\text { investigaciones } \\
\text { que consideren } \\
\text { cualquier tipo } \\
\text { de evaluación } \\
\text { de la capacidad } \\
\text { creativa en el } \\
\text { contexto de } \\
\text { ideas } \\
\text { emprendedoras. } \\
\text { Creatividad, } \\
\text { innovación y } \\
\text { reconocimiento } \\
\text { de las } \\
\text { oportunidades } \\
\text { dependen del } \\
\text { pensamiento } \\
\text { divergente; } \\
\text { El pensamiento } \\
\text { divergente } \\
\text { desarrolla } \\
\text { habilidades } \\
\text { cognitivas } \\
\text { esenciales para } \\
\text { los contextos } \\
\text { empresariales. }\end{array}$ \\
\hline
\end{tabular}




\begin{tabular}{|c|c|c|c|c|c|}
\hline & & & & $\begin{array}{l}\text { están relajados, } \\
\text { en otras } \\
\text { actividades } \\
\text { cotidianas que } \\
\text { no } \\
\text { directamente } \\
\text { involucran el } \\
\text { trabajo. }\end{array}$ & \\
\hline $\begin{array}{l}\text { Roels e } \\
\text { Van } \\
\text { Petege } \\
\text { m } \\
(2016)\end{array}$ & $\begin{array}{l}\text { Transdisciplinary } \\
\text { dimensions in } \\
\text { the composing } \\
\text { activities of } \\
\text { children: } \\
\text { Transfer of } \\
\text { strategies and } \\
\text { transformation } \\
\text { of knowledge. }\end{array}$ & $\begin{array}{l}\text { Analizar el } \\
\text { papel da } \\
\text { expresión visual } \\
\text { en la } \\
\text { composición } \\
\text { musical. }\end{array}$ & $\begin{array}{l}\text { Estudio de } \\
\text { casos } \\
\text { individuales } \\
\text { con abordaje } \\
\text { narrativa } \\
\text { descriptiva- } \\
\text { interpretativa. }\end{array}$ & $\begin{array}{l}\text { En un proceso } \\
\text { de orientación } \\
\text { el diálogo, los } \\
\text { nińos aprenden } \\
\text { a transformar } \\
\text { sus ideas (a } \\
\text { partir de la } \\
\text { reflexión sobre } \\
\text { los dibujos que } \\
\text { hacen) en } \\
\text { música; como } \\
\text { resultado } \\
\text { algunos niños } \\
\text { luego producen } \\
\text { composiciones } \\
\text { hechas } \\
\text { totalmente por } \\
\text { cuenta propia. } \\
\text { A la medida } \\
\text { que abstraen } \\
\text { sus dibujos e } \\
\text { historias, los } \\
\text { niños } \\
\text { construyen un } \\
\text { mundo interior } \\
\text { de sonido } \\
\text { extraído de su } \\
\text { imaginación. } \\
\text { Al } \\
\text { experimentar } \\
\text { una serie de } \\
\text { parámetros } \\
\text { musicales, } \\
\text { manipulan el } \\
\text { material de } \\
\text { acuerdo con } \\
\text { una } \\
\text { representación } \\
\text { visual de sus } \\
\text { ideas. }\end{array}$ & $\begin{array}{l}\text { Dibujar y } \\
\text { contar historias } \\
\text { permite que los } \\
\text { niños aprendan } \\
\text { a componer de } \\
\text { una forma más } \\
\text { holística. } \\
\text { Las actividades } \\
\text { de composición } \\
\text { están } \\
\text { inextricablemen } \\
\text { te conectadas a } \\
\text { otras áreas de la } \\
\text { actividad } \\
\text { musical. } \\
\text { Las estrategias } \\
\text { de composición } \\
\text { que los nińos } \\
\text { usan son más } \\
\text { una } \\
\text { confirmación } \\
\text { de la visión de } \\
\text { que del } \\
\text { pensamiento, } \\
\text { concebido } \\
\text { como } \\
\text { pensamiento } \\
\text { estructural, está } \\
\text { relacionado a } \\
\text { otras formas de } \\
\text { pensar, como o } \\
\text { pensamiento } \\
\text { narrativo, } \\
\text { matemático y } \\
\text { visual. } \\
\text { La capacidad de } \\
\text { los niños de } \\
\text { aprender y } \\
\text { comprender la } \\
\text { música en un } \\
\text { contexto trans e } \\
\text { interdisciplinar. }\end{array}$ \\
\hline
\end{tabular}

Fonte: Fonseca (2010), Guyotte et al. (2015), Keane e Keane (2016), LaDuca et al. (2019), Penaluna, Coates e Penaluna (2010), Roels e Van Petegem (2016)

En relación con el periodo de desarrollo, todos los artículos fueron publicados entre los anos de 2010 e 2019, ofreciendo contribuciones 
diferenciadas para proponer en las escuelas estrategias que colaboren para una cultura más justa y transdiciplinar.

Los artículos seleccionados tienen como punto común tratar educación y la transdisciplinariedad o bien, la educación y la justicia social, en diferentes áreas formativas, involucrando desde la enseñanza de música para niños (ROELS; VAN PETEGEM, 2016) a la formación de emprendedores creativos para atender a las demandas del mundo del trabajo (PENALUNA, COATES; PENALUNA, 2010). Al tratar del modelo STEAM (Ciencia, Tecnología, Ingeniería, Arte y Matemática), Guyotte et al. (2015, p. 15) destacan la intrínseca relación entre las herramientas transdisciplinarias en el pensamiento creativo: "La exploración de herramientas de pensamiento creativo transdisciplinar y un enfoque orientado a procesos para la resolución de problemas dio lugar a estudiantes con un lenguaje compartido para aventurarse en un espacio colaborativo de aprendizaje STEAM”.

Los objetivos de los estudios fueron significativamente diversificados, involucrando, desde la presentación de resultados de la implementación de una propuesta transdisciplinar de educación (FONSECA, 2010), vinculando diferentes asignaturas a través de un tema común y dentro de un espacio común, a la presentación de posibilidades para estimular el proceso de autonomía de los estudiantes en la búsqueda del conocimiento escolar y del mundo. También involucraran la presentación de pedagogías basadas en el diseño con destaque a abordajes cognitivas comprometidas con el desarrollo de mentalidades innovadoras, el análisis del papel de la expresión visual en la composición musical, además de la comprensión y la pertinencia social de la construcción de metodologías participativas y de creación de acontecimientos transdisciplinarios para el trabajo comunitario con jóvenes en el ámbito de la arte, la ciencia y la tecnología, bien como la descreación de una propuesta de 'STEAM by Design' como una iniciativa que desafía la mente de jóvenes con tareas comprometidas con un mundo mejor (KEANE; KEANE, 2016).

Metodológicamente, los estudios involucraram alternativas diversificadas, con predominio de estudios de caso (KEANE; KEANE (2016). También fueran realizadas revisiones de literatura (PENALUNA; COATES; PENALUNA, 2010) y descripción de experiencias (GUYOTTE et al., 2015). En relación con las técnicas e instrumentos de investigación fueran utilizados, entre otras alternativas, cuestionarios y entrevistas.

Considerando las especificidades de los objetivos, los resultados también indican múltiples posibilidades para crear alternativas educacionales comprometidas con una cultura más justa y transdisciplinar (GUYOTTE et al., 2015). Además de comprobar que colaborar en un ambiente 
transdisciplinar impacta significativamente las aspiraciones de carrera profesional de los estudiantes, llevó algunos de ellos a reconocer que la experiencia ha mejorado inclusive su capacidad de crear (LADUCA et al., 2019). Los estudios también comprobarán que: negocios y creatividad combinan, proporcionando una oportunidad para que los alumnos aprendan como innovar y ofrecer soluciones para problemas desafiadores; los clubes de arte, ciencia y tecnología son un ejemplo importante de escenarios educativos en que se articulan los afectos, las necesidades y los intereses de los jóvenes a partir de proyectos creativos; el foco en desafíos complejos de diseño de sustentabilidad local y global funcionan como un vehículo a través del cual fue posible pensar por medio de materiales, considerar el público y se involucrar con la comunidad; talleres con herramientas simultáneas para interacción con objetos, media, espacio y ambientes, realizados con estudiantes de la Enseńanza Fundamental y Media, generaran nuevas formas de aprender; la relevancia de los diálogos involucrando dibujos y canciones para que nińos aprendan a transformar sus ideas, siendo que a la medida que abstraían sus dibujos e historias, ellas contraían un mundo interior del sonido extraído de su imaginación.

En relación con las conclusiones, cabe destacar la relevancia de los estudios para: la involucración de estudiantes de diferentes asignaturas como oportunidad de compartir perspectivas con otras especialidades, además de estimular que la empatía influenciase en el producto por ellos generado y que la disponibilidad de un espacio flexible contribuyó para la colaboración.

\section{Discusión}

La diversidad de condiciones involucradas en los objetivos de las publicaciones seleccionas, entre ellas la vinculación de diferentes asignaturas, el uso de espacios comunes y el trabajo involucrando la comunidad, confirma la posibilidad de vinculación de la enseñanza a lo que Morin (2005) define como una sociedad compleja y permeada por una trama de condiciones que constituyen el mundo fenoménico. Tener en cuenta esa diversidad de condiciones en el currículo a partir de una perspectiva es una forma de promocionar el pensamiento complejo, estimulando la interacción con un conocimiento que para Sá (2019, p. 22) abarca a “[...] la multidimensionalidad del hombre, de la especie y de la sociedad [...]”.

La aproximación con la pregunta que nortea esta investigación sugiere que, para lograr, a través del pensamiento/competencia creativa desarrollada en las escuelas sociedades, una cultura más justa y transdiciplinar, es necesario 
mínimamente, pautar las prácticas transdisciplinares articuladas con posibilidades creativas como posible solución a problemas sociales reales, bien, con contribución del trabajo colaborativo, de la empatía, de la exploración de las tecnologías, y de la vinculación del aprendizaje con el arte.

Trabajar el contenido curricular en la interacción con parte substancial de los conceptos referenciados en las publicaciones (FONSECA, 2010; GUYOTTE ET AL., 2015; KEANE; KEANE, 2016; LADUCA et al., 2019; PENALUNA; COATES; PENALUNA, 2010; ROELS; VAN PETEGEM, 2016) es indispensable para la justicia escolar, siendo posible únicamente, para Rezende (2019), cuando se aproxima de las reales condiciones de los estudiantes. En este proceso, condiciones como el trabajo colaborativo, la empatía, el acceso a las tecnologías y la aproximación con los reales problemas sociales son especificidades indispensables para promocionar aquello que Estevão (2016, p. 53) define como dar “[...] más a los que más necesitan [...]. Destacar a su vez, que el proceso transdisciplinar y creativo es fundamental para que la educación contribuya con la justicia social, siendo además fundamental, el papel de los profesores creativos. Como ejemplo, se destaca el estudio de Henriksen (2012) en el que documenta las formas que el profesorado con éxito (finalistas del premio "National Teacher of the Year") actúan de forma creativa en sus clases. El autor investigó la forma como los profesores dinamizan las tareas en clase y llegó a la conclusión que transfieren activamente tendencias creativas de sus ocupaciones / intereses externos para sus prácticas de enseñanza.

Esa condición es estimulada en el Programa de Formación-Acción en Escuelas Creativas y fué observada por Zielinski (2019) en la investigación que acompañó el desarrollo de la propuesta en una alcaldía de Santa Catarina. Para esta autora, los docentes estimularan la superación de la enseńanza atomizada, por medio de actividades transdisciplinarias y ecoformadoras que aseguraran a involucrar en las clases de Educación Básica la realidad de propia ciudad, su cultura, su producción agrícola y otras especificidades locales mientras los alumnos estudiaban contenidos previstos en las diferentes asignaturas. De esa forma, ocurrió la transferencia de tendencias creativas de la realidad vivenciada fuera da escuela para la práctica pedagógica propuesta no contexto escolar.

$\mathrm{Al}$ promover reflexiones sobre la propia realidad de los estudiantes y de la población local de manera articulada al contenido escolar, la formación de los profesores ha creado la oportunidad para que, en la transferencia de tendencias creativas cotidianas para la clase, los docentes transitasen de prácticas disciplinares para propuestas transdisciplinares, indicando, que a 
pesar de ser un desafío, ese proceso constituí una posibilidad como afirman Almeida, Zwierewicz y Careño (2019).

Debe darse justicia social para que haya justicia escolar, por ello la formación docente necesita crear posibilidades para construir estrategias que estimulen a los estudiantes el permanecer en el sistema educativo, como defiende Rezende (2019). En el caso del Programa de Formación-Acción el Escuelas Creativas, la inclusión de la realidad local en las actividades propuestas en la escuela, conforme indicado por Zielinski (2019), favoreció el conocimiento complejo, o sea, el conocimiento pertinente y que para Sá (2019) abarca la vida en si y, por lo tanto, aquello que da sentido a las actividades desarrolladas por los estudiantes en el contexto escolar.

Por otro cabo, es importante remarcar la relevancia de investigaciones que involucren: la evaluación de la capacidad creativa en el contexto de ideas emprendedoras (PENALUNA; COATES; PENALUNA, 2010); la formación de los profesionales comprometidos con el contexto social (GUYOTTE et al., 2015); la invención de rutas metodológicas para activar procesos de formación interdisciplinarios, generar formas de pensamiento y de abstracción frente a lo que se piensa y se hace (FONSECA, 2010); el uso de recursos online para expandir las oportunidades de diseño y construir informaciones, tomando decisiones que permitan procesar nuevas informaciones a lo largo del tiempo (KEANE; KEANE, 2016); organizar diferentes asignaturas para compartir perspectivas con otras especialidades (LADUCA et al., 2019).

\section{Conclusiones}

La revisión de los artículos disponibles en diferentes bases de datos abordando formas de lograr una cultura más justa por medio de una enseñanza transdisciplinar posibilitó identificar diferentes conceptos, estrategias y recursos que posibilitan ese proceso. Caminos estos, que superan una visión descontextualizada y atomizada de práctica pedagógica y apuntan para la relevancia de evitar actividades centradas en el aislamiento de los estudiantes, además de la emergencia de la emergencia de proponer nuevos escenarios para el aprendizaje.

Además, al valorizar la interacción entre las asignaturas y de las mismas en relación con la realidad de los estudiantes, observase la relevancia de la transdisciplinariedad. Cuando en la práctica pedagógica se dialoga con diferentes realidades sociales y con las emergencias globales, como la cuestión de la sostenibilidad, se incorpora un nuevo sentido para los procesos de enseńanza y aprendizaje, vinculado a la pertinencia del conocimiento y el 
sentimiento de pertinencia de los estudiantes, pues se trata del estudio de conocimientos vivos.

Destacarse también el énfasis la flexibilidad de las estrategias didácticas y el estímulo a la empatía constituyen alternativas indispensables para una enseñanza conectada con las demandas de la sociedad actual, sean ellas locales o globales. De la misma forma, el desarrollo de estudios en diferentes escenarios de aprendizaje y el estímulo a la colaboración son determinantes para la capacidad de enfrentamiento de las adversidades que los estudiantes necesiten superar, especialmente en este momento de la historia en que las incertidumbres delante el futuro de la humanidad son más expresivas que la seguridad de un futuro promisor para grande parte de la población mundial.

Son las condiciones contextualizadas en este artículo las que proponemos, pueden subsidiar iniciativas desde la investigación (elementos de la revisión sistemática) y desde la acción (misión y visión de la red de escuelas creativas) que se comprometan con la perspectiva educacional de la práctica educativa. En ese sentido, cabe destacar que dichas propuestas deben ir acompañadas de la formación del profesorado, con el fin de facilitar la implementación de encuentros y vivencias creativas significativas.

\section{Referências}

ADRA, Suzy. The states of presence and insight in the painting process. Dissertation Abstracts International Section A. Humanities and Social Sciences, [s. 1.], v. 77, n. 11-A-E, 2017. Disponible en: http://sire.ub.edu/login?url=http://search.ebscohost.com/login.aspx?. Acceso en: 12 ago. 2020.

AMMAR, Achraf et al. Effects of COVID-19 home confinement on physical activity and eating behavior. Preliminary results of the ECLB-COVID19 international online-survey. Medrxiv, [s. l.], v. 12, n. 6, p. 1-23. 2020. DOI: https://doi.org/10.1101/2020.05.04.20072447. Disponible en: https://www.medrxiv.org/content/10.1101/2020.05.04.20072447v1.full.pd f. Acceso en: 23 jul. 2020.

ALMEIDA, Aline Lima da Rocha Almeida. Influência do Programa de Formação-Ação em Escolas Criativas na transformação das práticas pedagógicas em uma escola do campo. Dissertação (Mestrado Profissional em Educaçáo Básica) - Universidade Alto Vale do Rio do Peixe, Caçador, 2018. 
ALMEIDA, Aline Lima da Rocha Almeida; ZWIEREWICZ, Marlene; CARREÑO, Leticia. Do Programa de Formação-Ação em Escolas Criativas ao planejamento transdisciplinar e ecoformador em uma Escola do campo. Revista Extensão em Foco, Caçador, v. 7, n. 2, p. 91-107, 2019. Disponible en: https://periodicos.uniarp.edu.br/index.php/extensao/article/view/2164. Acceso en: 20 jul. 2020.

ASSOCIATION FOR THE ADVANCEMENT OF COMPUTING IN EDUCATION. E-Learn 2012. World Conference on E-Learning in Corporate, Government, HEALTHCARE \& HIGHER EDUCATION. PROCEEDINGS, 1, Montreal. Anais [...]. Montreal: Association for the Advancement of Computing in Education, 2012. p. 9-12.

AYVAZ, Meliha Dzirlo. Generating creativity in teams: an exploratory case study. The George Washington University, Human \& Organizational Learning, US. Dissertation Abstracts International Section A: Humanities and Social Sciences, [s. l.], v. 79, n. 1-A-E, p. 1-117, 2018. Disponible en: https://search.proquest.com/openview/55e638c57c2d1e553b25ccac0d262ac 3/1 ?pq-origsite=gscholar\&cbl=18750\&diss=y. Acceso en: 12 ago. 2020.

CAMACHO-CARDENOSA, Alba et al. Influencia de la actividad física realizada durante el confinamiento en la pandemia del COVID-19 sobre el estado psicológico de adultos: un protocolo de estudio. Rev Esp Salud Pública, [s. l. $] \quad$ v. 94, p. 1-9, 2020. Disponible en: https://medes.com/publication/151919. Acceso en: 10 ago. 2020.

CHARYTON, Christine. Creativity and innovation among science and art: A discussion of the two cultures. New York: Springer-Verlag Publishing; 2015.

DEHNAD, Vida. Sustainable Transdisciplinary Future for English Majors in Iran by Implementing a New Paradigm. Interchange: A Quarterly Review of Education, [s. l.], v. 50 n.1 p. 77-96, feb. 2019. DOI: https://doi.org/ Disponible en: https:/link.springer.com/article/10.1007/s10780-0189342-5. Acceso en: 12 ago. 2020.

DEPALO, Ralph; BRENNAN, Mark. Spiritual Caregiving for Older Adults: A Perspective from Clinical Practice. Journal of Religion, Spirituality \& Aging, [s. l.], v. 17, n. 1-2, p. 151-160, 2004. DOI: https://doi.org/10.1300/J496v17n01_08. Disponible en: https://www.researchgate.net/publication/233276935_Spiritual_Caregiving _for_Older_Adults_A_Perspective_from_Clinical_Practice. Acceso en: 10 ago. 2020 . 
ESTEVÃO, Carlos V. Justiça social e modelos de educação: para uma escola justa e de qualidade. Revista Diálogo Educacional, Curitiba, v. 16, n. 47, p. 3758, 2016. DOI: http://dx.doi.org/10.7213/dialogo.educ.16.047.DS02. Disponible en: https://periodicos.pucpr.br/index.php/dialogoeducacional/article/view/1903 . Acceso en: 6 abr. 2020.

FONSECA, Andrés. Metodologías participativas, subjetividades expandidas y transdisciplinariedad. Tesis Psicológica, [s. l.], n. 6, v. 1, p. 52-73, 2010. Disponible en: https://revistas.libertadores.edu.co/index.php/TesisPsicologica/article/view/2 45. Acceso en: 6 ago. 2020.

GUIRAO-GORIS, Silamani J. A. Utilidad y tipos de revisión de literatura. ENE, Revista de Enfermería, [s. l.], v. 9, n. 2, p. 2-13, 2015. Disponible en: https://www.researchgate.net/publication/281449479_Utilidad_y_tipos_de _revision_de_la_literatura. Acceso en: 10 ago. 2020.

GUIRAO-GORIS, Silamani J. A; OLMEDO SALAS, Angela; FERRER FERRANDIS, Esperanza El artículo de revisión. Revista Iberoamericana de Enfermeria Comunitaria, [s. l.], v. 1, n 1, p. 1-25, 2008. Disponible en: https://www.researchgate.net/publication/268518751_El_articulo_de_revisi on. Acceso en: ago. 2020.

GUYOTTE, Kelly W. et al. Steam as Social Practice: Cultivating Creativity in Transdisciplinary Spaces. Art Education, [s. l.], v. 67, n. 6, p 12-19, nov. 2014. Disponible en: http://www.arteducators.org/research/art-education. Acceso en: 28 ago. 2020.

GÜLERCE, Aydan. Conceptual and conditional (im)possibilities of creative theorizing of creativity and culture: Critical reflections from Turkey toward globally transformative praxis. In: GLFLVEANU, Vlad Petre (ed.). The Palgrave handbook of creativity and culture research. New York: Palgrave Macmillan/Springer Nature, 2016. p. 425- 448.

HALL, Kara L. et al. Moving the science of team science forward: Collaboration and creativity. American Journal of Preventive Medicine, [s. l.], v. 32, n. 2, suplemento 1, p. S243-S249, ago., 2008. DOI: https://doi.org/10.1016 / j.amepre.2008.05.007. Disponible en: https://pubmed.ncbi.nlm.nih.gov/18619406/. Acceso en: 10 ago. 2020.

HAUK, Marna. Gaia e/mergent: Earth regenerative education catalyzing empathy, creativity, and wisdom. Dissertation Abstracts International Section 
A: Humanities and Social Sciences, [s. l.], v. 75, n. 11-A-E, p. 1-668, 2015. Disponible en: https://pqdtopen.proquest.com/doc/1563382491.html?FMT=ABS. Acceso en: 10 ago. 2020.

HELMICK, Linda J. Extraordinary expressions of trust and vulnerability: An arts-informed transdisciplinary journey through blended landscapes of art education and art therapy. Bloomington: Universidad de Indiana, 2019.

HENRICKSON, Jeni Kathryn. Adventure, inquiry, and technology as driving forces in sustainability education. Dissertation Abstracts International Section A: Humanities and Social Sciences, [s. l.], v. 77, n. 12-A-E, p. 1-158, 2016. Disponible en: https://conservancy.umn.edu/handle/11299/182280. Acess Acceso en: 10 ago. 2020.

HENRIKSEN, Danah. We teach who we are: Creativity and transdisciplinary thinking in the practices of accomplished teachers. Dissertation Abstracts International Section A: Humanities and Social Sciences, [s. l.], v. 73, v. 4-A, -. 1-227, 2012. Disponible en: http://danah-henriksen.com/wpcontent/uploads/2013/06/Danah-Dissertation.pdf. Acceso en: 10 ago. 2020.

JACKSON, Lesley A. The implications of self-creation and self-care in higher education: A transdisciplinary inquiry. Dissertation Abstracts International Section A. Humanities and Social Sciences, [s. l.], v. 79, n. 1-A-E, p. 1-227, 2018 . Disponible en: https://eric.ed.gov/?id=ED578937. Acceso en: 10 ago. 2020.

KAHHALE, Edna Maria Peters; ESPER, Elisa Maria Barbosa. Novas possibilidades metodológicas: a quebra dos paradigmas qualitativo e quantitativo em psicologia. Psicologia \& Sociedade, [s. l.], v. 26, esp. p. 70-83, 2014. DOI: https://doi.org/10.1590/S0102-71822014000600008. Disponible en: https://www.scielo.br/scielo.php?pid=S010271822014000600008\&script=sci_abstract\&tlng=pt. Acceso en: 9 ago. 2020.

KEANE, Linda; KEANE, Mark. STEAM by Design. Design and Technology Education: An International Journal, [s. l.], v. 21, n. 1. p. 61-82, fev., 2016. Disponible en: https://ojs.lboro.ac.uk/DATE/article/view/2085. Acceso en: 12 ago. 2020.

LADUCA, Brian et al. A Transdisciplinary Collaboration and Innovation Education Model and Experience. Research in Higher Education Journal, [s. 
l.], v. 37, p. 1-31, nov 2019. Disponible en: https://eric.ed.gov/contentdelivery/servlet/ERICServlet? accno=EJ1233129. Acceso en: 28 ago. 2020.

MARCHIORI, Marlene; OLIVEIRA, Ivone. Perspectives, challenges, and future directions for organizational communication research in Brazil. Management Communication Quarterly, [s. l.], v, 22, n. 4, p. 671-676, mayo, 2009. DOI: https://doi.org/10.1177 / 0893318909332070 . Disponible en: https:/www.researchgate.net/publication/238334236_Perspectives_Challen ges_and_Future_Directions_for_Organizational_Communication_Research _in_Brazil. Acceso en: 10 ago. 2020.

MERCIECA, Daniel. 'I'm not so sure, Miss'. The concept of uncertainty and dramatherapy practice within the context of transdisciplinary work in an educational setting. In: LEIGH, Lauraine et al. (ed). Dramatherapy with children, young people and schools: Enabling creativity, sociability, communication and learning. New York: Routledge/Taylor \& Francis Group, 2012. p. 145-155.

MOHER, David et al. Preferred Reporting Items for Systematic Reviews and Meta-Analyses: The PRISMA Statement. PLoS Medicine, [s. l.], n. 6, v. 6, p. 1-6, 2009. DOI: https://doi.org/10.1371/journal.pmed.1000097. Disponible en: https://journals.plos.org/plosmedicine/article?id=10.1371/journal.pmed.100 0097. Acceso en: 17 jul. 2020.

MONTUORI, Alfonso; DONNELLY, Gabrielle. The creativity of culture and the culture of creativity research: The promise of integrative transdisciplinarity. In: GLFLVEANU, Vlad Petre (ed.). The Palgrave handbook of creativity and culture research. New York: Palgrave Macmillan/Springer Nature, 2016. p. 743- 765.

MORIN, Edgar. Ciência com Consciência. 2. ed. Rio de Janeiro: Berhand, 1998.

MORIN, Edgar. Introdução ao pensamento complexo. Porto Alegre: Sulina, 2005.

MORIN, Edgar. Ensinar a viver: manifesto para mudar a educação. Tradução de Edgard de Assis Carvalho e Mariza Perassi Bosco. Porto Alegre: Sulina, 2015. 
PICUCCI, Michael. Ritual as resource: Health and transformation in the twenty-first century. In: GORDON-LENNOX, Jeltje (ed); Emerging ritual in secular societies: A transdisciplinary conversation. London: Jessica Kingsley Publishers, 2017. p. 140-157.

PRATER, Greg. New Hopes, New Horizons: The Challenges of Diversity in Education. INTERNATIONAL ASSOCIATION OF SPECIAL EDUCATION, 5, 1997, Anais [...]. Capetown: International Association Of Special Education, 1997. p. 3-8.

PENALUNA, Andrew; COATES, Jackie; PENALUNA, Kathryn. CreativityBased Assessment and Neural Understandings: A Discussion and Case Study Analysis Education \& Training, [s. l.], v. 52, n. 8-9, p. 660-678, 2010. Disponible en: http://www.emeraldinsight.com.sire.ub.edu. Acceso en: 28 ago. 2020.

PETRAGLIA, Izabel. Pensamento complexo e educação. São Paulo: Livraria da Física, 2013.

REASON, Matthew et al. Spectators' aesthetic experience of sound and movement in dance performance: A transdisciplinary investigation. Psychology of Aesthetics, Creativity, and the Arts, [s. l.], v. 10, n.1, p. 42-55. feb, 2016. DOI: https://doi.org/https://doi.org/10.1037/a0040032. Disponible en: https://psycnet.apa.org/record/2016-06979-003. Acceso en: 10 ago. 2020.

REGO, Sergio Tavares de Almeida; PALÁCIOS, Marisa. Ética e democracia em tempos de crise. Saúde debate, [s. l.], v. 40, p. 63-73, 2016. DOI: http://dx.doi.org/10.1590/0103-11042016s06. Disponible en: https:/www.scielo.br/scielo.php?pid=S0103-

11042016000500063\&script=sci_abstract\&tlng=pt. Acceso en: 12 ago. 2020.

REGO, Sergio Tavares de Almeida; PALÁCIOS, Marisa. Justiça social como um imperativo ético. Saúde Debate, Rio de Janeiro, v. 43, n. esp. 7, p. 141151, dez., 2019. DOI: http://dx.doi.org/10.1590/0103-11042019S711. Disponible en: https://www.scielo.br/scielo.php?pid=S0103$11042019001200141 \&$ script=sci_abstract\&tlng=pt. Acceso en: 18 jul. 2020.

REZENDE, Maria José de. Justiça escolar e justiça diferencialista nos documentos adotados por órgãos das Nações Unidas (PNUD E UNESCO). Lua Nova, Revista de Cultura e Política, São Paulo, n. 108, p. 121-135, 2019. DOI: https://doi.org/10.1590/0102-121135/108. Disponible en: 
https://www.scielo.br/scielo.php?script=sci_arttext\&pid=S010264452019000300121. Acceso en: 8 ago. 2020.

ROSENCRANS, Kendra. Narratives of ingenuity: Using coworking space stories to see systems change. Dissertation Abstracts International: Section B: The Sciences and Engineering, [s. l.], v. 81, n 2-B, p. 1-184, 2020. Disponible en: https://search.proquest.com/openview/31eda70976423bdda627fed6b4ef28 16/1 ?pq-origsite=gscholar\&cbl=18750\&diss=y. Acceso en: 10 ago. 2020.

ROELS, Johanna Maria; VAN PETERSON, Peter. Transdisciplinary dimensions in the composing activities of children: transfer of strategies and transformation of knowledge. British Journal of Music Education, [s. l.], v. 33, n. $1, \quad$ p. $81-99, \quad$ mar, 2016. DOI: https://doi.org/10.1017/S026505171500025X. Disponible en: ambridge.org/core/journals/british-journal-of-musiceducation/article/transdisciplinary-dimensions-in-the-composing-activitiesof-children-transfer-of-strategies-and-transformation-ofknowledge/9D091BDCBF1C2886A7F8EDD. Acceso en: 5 ago. 2020.

SÁ, Ricardo Antunes. Contribuições teórico-metodológicas do pensamento complexo para a construção de uma pedagogia complexa. In: SÁ, Ricardo Antunes; BEHRENS, Marilda Aparecida (org.). Teoria da complexidade: contribuiçóes epistemológicas para uma pedagogia complexa. Curitiba: Appris, 2019. p. 17-64.

STROEBE, Wolfgang. Interdisciplinary Research and Creativity. Utrecht University, Utrecht, In: VAN LANGE, Paul A. M. (ed). Netherlands Bridging social psychology: Benefits of transdisciplinary approaches. Mahwah: Lawrence Erlbaum Associates Publishers; 2006. p. 71-76.

THOMPSON, Tess; KREUTER, Matthew. Using written narratives in public health practice: A creative writing perspective. Preventing Chronic Disease: Public Health Research, Practice, and Policy, [s. l.], v. 11, p. 1-7, jun 5, 2014. DOI: https://doi.org/0.5888/pcd11.130402. Disponible en: https:/www.ncbi.nlm.nih.gov/pmc/articles/PMC4049201/. Acceso en: 10 ago. 2020.

TORRE, Saturnino de la. Instituciones educativas creativas: instrumento para valorar el desarrollo creativo de las Instituciones Educativas (VADECRIE). El Ejido: Circulo Rojo, 2012a . 
TORRE, Saturnino de la (cor.). Creadores en la adversidad: relatos admirables para tiempos de crisis ¿rebeldes con causa? El Ejido: Circulo Rojo, 2012b.

TORRE, Saturnino de la. Escolas criativas: escolas que aprendem, criam e inovam. In: ZWIEREWICZ, Marlene; TORRE, Saturnino de la. (org.). Uma escola para o século XXI: escolas criativas e resiliência na educação. Florianópolis: Insular, 2009. p. 55-69.

TORRE, Saturnino de la. Movimento de escolas criativas: fazendo parte da história de formação e transformação. In: ZWIEREWICZ, Marlene (org.). Criatividade e inovação no Ensino Superior: experiências latino-americanas e europeias em foco. Blumenau: Nova Letra, 2013. p. 139-162.

VAUGHAN, James et al. Developing creativity to enhance human potential in sport: A wicked transdisciplinary challenge. Frontiers in Psychology, [s. l.], v. 10, p. 1-16, sep. 13, 2019. DOI: https://doi.org/https://doi.org/10.3389/fpsyg.2019.02090. Disponible en: https://www.frontiersin.org/articles/10.3389/fpsyg.2019.02090/full. Acceso en: 10 ago. 2020.

VICENT, María et al. Perfectionism profiles and motivation to exercise based on Self-Determination Theory. International Journal of Environmental Research and Public Health, [s. l.], v. 17, n. 9, p. 1-12, 2020. DOI: https://doi.org/10.3390/ijerph17093206.

Disponible en: https://www.ncbi.nlm.nih.gov/pmc/articles/PMC7246553/. Acceso en: 25 jul. 2020.

WHITBREAD, Richard Guy. Cultural and arts education policies in Hong Kong: Two wings of the same bird? Dissertation Abstracts International Section A: Humanities and Social Sciences, [s. l.], v. 78, n. 7-A-E, p. 1-401, 2018Disponible en: http://libdr1.ied.edu.hk/pubdata/img00/arch00/link/archive/1/instarh/4378 _image.pdf. Acceso en: 10 ago. 2020.

WEST, Michael A. Dissent in Teams and Organizations: Lessons for Team Innovation and Empowerment. In: VAN LANGE, Paul A. M. (ed); Bridging social psychology: Benefits of transdisciplinary approaches. Mahwah: Lawrence Erlbaum Associates Publishers, 2006. p. 353-358.

ZIELINSKI, Helena Castilho. Indicadores de práticas transdisciplinares de leitura, produção e interpretaçáo textual detectados no Programa de FormaçãoAção em Escolas Criativas. 2019. Dissertação (Mestrado Profissional em Educação Básica) - Universidade Alto Vale do Rio do Peixe, Caçador, 2019. 
ZWIEREWICZ, Marlene; TORRE, Saturnino de la. (org.). Uma escola para o século XXI: Escolas Criativas e resiliência na educação. Florianópolis: insular, 2009.

ZWIEREWICZ, Marlene. Projetos Criativos Ecoformadores - PCE: inovação metodológica e estímulo à transdisciplinaridade no Ensino Superior. In: ZWIEREWICZ, Marlene (org). Criatividade e inovação no Ensino Superior: experiências latino-americanas e europeias em foco. Blumenau: Nova Letra, 2013. p. 136-186.

ZWIEREWICZ, Marlene. Programa de Formação-Ação em Escolas Criativas: matizes da pedagogia ecossistêmica na formação de docentes da Educação Básica. In: DITTRICH, Maria Gloria et al. (org.). Políticas Públicas na contemporaneidade: olhares cartográficos temáticos. Itajaí: Univali, 2017. p. 217-231.

Recebido em: 13 ago. 2020

Aceito em: 25 ago. 2020 